antibodies (LGI1, CASPR2, DPPX, MOG, Aquaporin4, GQ1b, DPPX, MOG, Aquaporin4, and GQ1b) as opposed to intracellular antibodies.

In conclusion, FDG-PET may provide additional supportive data to aid early diagnosis of AE especially in the context of normal MRI imaging or negative antibody testing. It may also prove useful in monitoring the effect of treatment.

\section{G60(P) RED FLAGS: PARENT-REPORTED EARLIEST CONCERNS REGARDING THEIR CHILD'S EMERGING CEREBRAL PALSY}

IJ Baggaley, ${ }^{2} \mathrm{~T}$ Rapley, ${ }^{1,3} \mathrm{~A}$ Basu. ${ }^{1}$ Institute of Health and Society, Newcastle University, Newcastle Upon Tyne, UK; ${ }^{2}$ Department of Social Work, Education and Community Wellbeing, Northumbria University, Newcastle Upon Tyne, UK; ${ }^{3}$ Department of Paediatric Neurology, Great North Children's Hospital, Newcastle Upon Tyne, UK

\subsection{6/archdischild-2020-rcpch.46}

Background Each year around 1800 UK children are diagnosed with Cerebral Palsy (CP). Of these, $40-50 \%$ are deemed 'low risk' at birth and therefore rely on their parents and primary care health professionals (HCPs) to identify concerning features and seek early referral. Some parents report difficulties in communicating their concerns, and being falsely reassured.

Aims To describe the nature of the earliest concerns parents report to HCPs in children with emerging CP.

Methods Parents of children with CP were recruited via social media to take part in an, ethically approved, online survey. This collected information on child and respondent demographics and the earliest concerns parents had regarding their child's development. Thematic analysis was undertaken on the first 200 responses.

Results Four responses were excluded due to ineligibility. Most responses $(n=186)$ were from mothers. Children with Hemiplegic CP made up $51.5 \%$ of the sample (table 1). Eight main themes emerged; Developmental Milestones $(n=141)$, Atypical Movements ('His left leg did not kick excitedly in the bath') $(n=97)$, Worrying Birth History $(n=86)$, Posture $(n=69)$, Tone $(n=48), \quad$ Feeding $(n=23), \quad$ Temperament (including sleep) $(n=22)$, and Parental Intuition ('I just knew something wasn't right.') $(\mathrm{n}=16)$.

Conclusion/Discussion Developmental Milestones were the most frequently reported concern, supporting current screening processes. However, this represented only $28 \%$ of the total concern burden. Although some concerns were non-specific, a proportion of concerns in all categories were not acted on.

\begin{tabular}{llllllll} 
Abstract G60(P) Table 1 & Participants CP Type and GMFCS score \\
\hline Typography & $<2$ years of age & 1 & 2 & 3 & 4 & 5 & Total \\
\hline & - & - & 1 & - & - & - & 1 \\
\hline Monoplegia - Upper Limb & - & - & - & 2 & 1 & 2 & 5 \\
Monoplegia - Lower Limb & - & 1 & 3 & 7 & 35 & 47 & 101 \\
Hemiplegia & 8 & - & 3 & 10 & 4 & 2 & 20 \\
Diplegia & 1 & - & 2 & 10 & 2 & 3 & 17 \\
Triplegia & - & 25 & 12 & 5 & 5 & 1 & 52 \\
Quadriplegia & 4 & 26 & 21 & 34 & 47 & 55 & 196 \\
Total & 13 & & & & &
\end{tabular}

Limitations Retrospective nature; lack of clinician confirmation of clinical details.

Strengths Potential to further mine responses to identify parental concerns not acknowledged by HCPs, as potential new 'red flags'. Such data could improve early recognition and referral of infants with emerging CP.

\section{G61(P) WHAT SHOULD TRUSTS CHARGE NHS ENGLAND TO DELIVER SPECIALIST INPATIENT NEUROREHABILITATION IN THE SETTING OF A REGIONAL PAEDIATRIC NEUROSCIENCE CENTRE?}

${ }^{1}$ PM Sharples, ${ }^{2} \mathrm{~L}$ Shuyu, ${ }^{1} \mathrm{~A}$ Spray, ${ }^{2} \mathrm{HK}$ Smith. ${ }^{1}$ Bristol Royal Hospital for Children, UHBT, Bristol, UK; ${ }^{2}$ CORMSIS team, University of Southampton, Southampton, UK

\subsection{6/archdischild-2020-rcpch.47}

Aims To calculate what bed-day tariff a paediatric Regional Neuroscience Centre (RNSC) should charge to deliver a quality, sustainable specialist in-patient neurorehabilitation service, taking into account patient complexity/dependency.

Methods Ethical approval was obtained from University of Southampton. Anonymized data on children receiving inpatient neurorehabilitation at a paediatric RNSC 1/6/2017$31 / 5 / 2019$ were analyzed. Patient dependency and rehabilitation complexity was assessed by Rehabilitation Complexity Scale (RCS); this provides an overall measure of Care or Risk, Nursing, Therapy, Medical and Equipment needs. RCS-Extended (version-13) was scored for neurorehabilitation patients jointly by multi-disciplinary team members at weekly clinical meetings. RCS-E scores were categorized as: very low level of rehabilitation need (0-4); low level (5-8); medium level (9-12); high level (13-16); very high level (17-22). Discrete-Event Simulation (DES) was used to simulate in-patient neurorehabilitation service work flow, calculating how many patients would be treated each day and their changing rehabilitation needs. Staffing numbers required were based on the modelling results, applying published minimum staffing provisions for adult neurorehabilitation centers. Service costs and bed-day tariffs were calculated from calculated staff numbers, assuming 25\% overheads.

Results The database contained 114 children who received inpatient neurorehabilitation over the two-year study period. 21 had no RCS data; 29 others were admitted before the study period, 4 discharged afterwards. Excluding these patients, 69 were used in the model, which mapped routes between dependency levels. Modelling showed 91 patients to be admitted and discharged by the neurorehabilitation service over two years (95\% CI 75-107). Mean Length of Stay $=53$ days $(95 \%$ CI 44-62). Mean number of beds occupied at any one time by neurorehabilitation in-patients $=6.3(95 \% \mathrm{CI} 4-8-8.0)$. Of these, 0.65 (95\% CI $0.17-1.26$ ) were occupied by patients with very high needs; 1.73 (CI 1.08-2.45) high needs; 2.25 (CI 1.58-291) medium needs; 0.20 (CI $0.13-0.28$ ) low needs; 0.0 .8 (CI $0.02-0.17)$ very low needs. Applying differential published adult staffing standards to the modelled complexity/dependency levels and assuming 25\% overheads, the bed-day tariff required to support the specialist neurorehabilitation in-patient service ranged from $£ 622.45-£ 754.75$.

Conclusions Application of simulation modelling to real-world paediatric data suggests costs in keeping with those charged by adult centers. 